\title{
Prescription Pattern And Potential Drug- Drug Interactions of Patients Hospitalised For Urinary Tract Infection in A Tertiary Care Hospital
}

\author{
Pratibha omkar, Padmaja Udaykumar \\ Department of Pharmacology Father Muller Medical college and hospital, Mangalore- 575002, Karnataka, \\ India
}

\begin{abstract}
Urinary tract infections (UTI) are quite common in the community medical practice. The objective of our study was to know the prescription pattern of drugs used in the treatment of UTI. A retrospective observational study was conducted at Father Muller Medical College, Mangalore. The case records of 150 patients of either sex who were admitted to Father Muller college and hospital between May 2015 to April 2016 with diagnosis of UTI were studied. The incidence of UTI was more in females .Majority of patients were treated with antibiotics. Cephalosporins were most commonly prescribed group of antimicrobial agent followed by fluroquinolones.E.coli was the most common uropathogen isolated. Of 244 potential drug interactions noted, $54 \%$ were pharmacokinetic in nature.
\end{abstract}

Keywords: UTI, Antibiotics, Prescription, Cephalosporins, Fluroquinolones, E.coli

\section{Introduction}

Urinary tract infection (UTI) is defined as the presence of bacteria in urine along with symptoms of infection. It is one of the most common infections described in outpatients setting.It occurs mainly in women without underlying diseases and with no functional or structural anomalies of the urinary tract; consequently most cases are considered uncomplicated UTI. The majority of UTIs are caused by Escherichia coli (70-95\%), Proteus mirabilis, Klebsiella spp. and Staphylococcus saprophyticusaccounting for 1-2\%, 1-2\% and 5-10\% of infections respectively. ${ }^{1}$

Urinary tract infections are common burden in patients with diabetes mellitus. Cystitis, ascending infection leading to pyelonephritis, impaired leucocyte function, recurrent vaginitis, emphysematous complications and renal/perinephric abscesses are well recognized in this group of patients if glycemic control is poor. Infections particularly in pregnancies and in the elderly can be asymptomatic, but asymptomatic bacteriuria is associated with an increased risk of intrauterine growth retardation and low birth weight babies. Furthermore, untreated asymptomatic bacteriuria leads to development of cystitis in approximately $30 \%$ of cases, and can lead to the development of pyelonephritis in about $50 \%$ of cases. Thus it is important to identify and treat UTI to avoid such complication. ${ }^{2}$

Potential Drug-drug interactions (PDDI) in patients receiving multidrug therapy is a major concern. Drug-drug interactions (DDIs) are defined as two or more drugs interacting in such a manner that the effectiveness or toxicity of one or more drugs is altered ${ }^{3}$.Drug interactions may lead to an increased risk of hospitalization and higher health care costs. The incidence of actual occurrence of drug interactions has been reported to be ranging from 0 to $1.3 .^{4} \mathrm{~A}$ rational and informed approach to drug interactions, based on scientific knowledge, can reduce the chance of adverse effects and improve patient outcomes. ${ }^{4}$ Hence the present study was undertaken to find out the prescription pattern, common uropathogens responsible for UTI, and drug- drug interactions in the patients.

\section{Objectives}

1. To evaluate prescription pattern of UTI in a tertiary care hospital.

2. To study major symptoms and most commonuropathogens causing UTI.

3. To study the potential drug-drug interactions in patients who are on treatment.

\section{Materials And Methods}

The study was conducted at Father Muller Medical College, Mangalore. This wasa retrospective observational study which included all inpatients admitted to Father Muller hospital with a diagnosis of UTI between May 2015 to April 2016. The case record files were retrieved from the medical records department based on the ICD- 10 disease coding (World Health Organization, ICD-10, F00-F99).The demographics, dose, frequency and duration of drugs used to treat the urinary tract infection of 150 files were recorded. The relevant investigations (microbiological and haematological) were noted down and the potential drug interactions 
wereanalyzed by using the online freely available drug interaction checker.(Medscape reference interaction checker)

\section{Mode of data analysis:}

The mode of data analysis was done with respect to age distribution, sex ratio, frequency of different classes of drugs used, antibiotics utilization pattern, potential drug interactions among the drugs prescribed.

\section{Inclusion criteria :}

Patients receiving drug therapy in UTI of either gender of age $>18$ years, ( UTI in pregnant women also ) were included in this study.

\section{Exclusion criteria :}

Patients of $<18$ years of either gender and asymptomatic UTI were excluded from the study

Study design :

Retrospective observational study was done to assess the prescription pattern in UTI in a tertiary care hospital.

Sample size -150

\section{Method of data collection}

A cross sectional retrospective study was conducted in June 2016 for a period of 1 month. The case record files will be retrieved from the medical records department based on the ICD-10 disease coding (World health organization, ICD-10, F00-F99).

\section{Statistical analysis}

Data was entered in Microsoft Excel sheet and analysed by mean, standard deviation, frequency and percentage. Drug interactions were checked through the Medscape drug interaction checker.

\section{Results}

A total number of patients enrolled in the study were 150, of which $66 \%$ were females and $34 \%$ males. (fig.1). Majority of patient were seen in the age group of 31-45 years.(table.1)

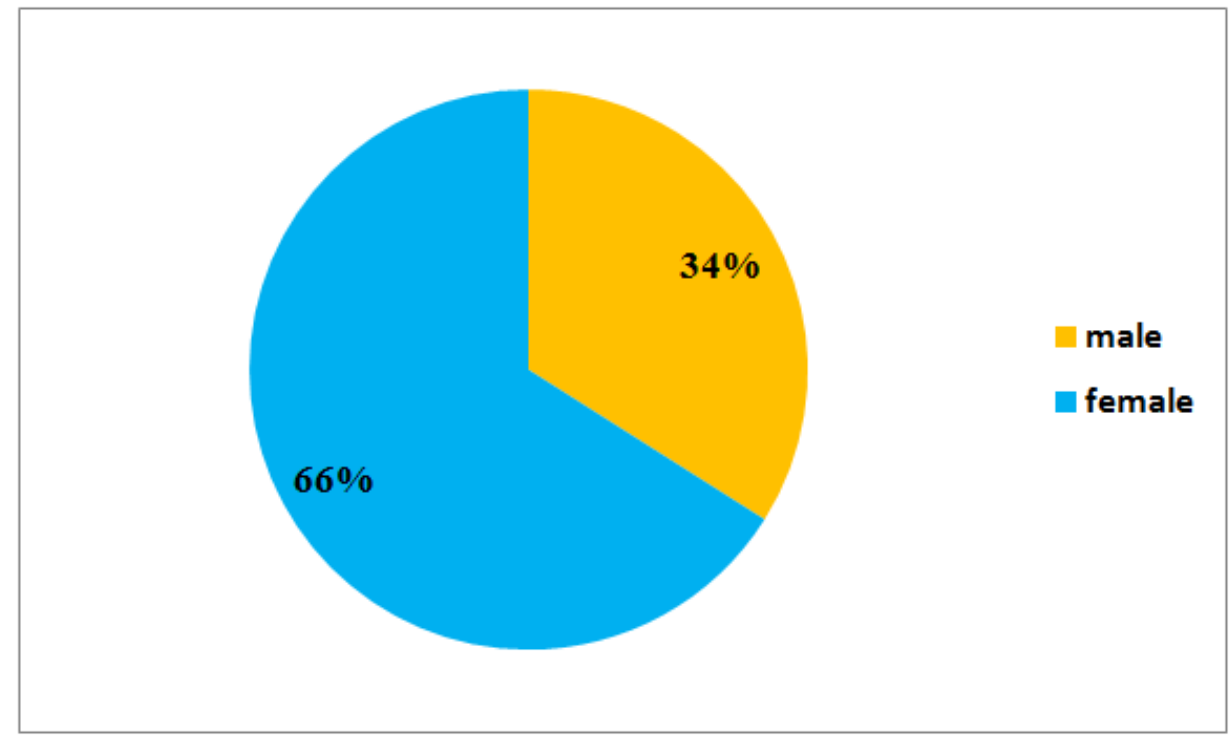

Figure 1. Pie chart depicting the gender distribution

\begin{tabular}{|l|l|l|}
\hline AGE & Frequency & Percentage \\
\hline $1-\quad 18$ years & 23 & $15.3 \%$ \\
\hline 19-30years & 45 & $30 \%$ \\
\hline 31-45 years & 35 & $23.3 \%$ \\
\hline 46- 60 years & 35 & $23.3 \%$ \\
\hline$>60$ years & 12 & $8 \%$ \\
\hline Total & 150 & $100 \%$ \\
\hline
\end{tabular}

Table 1.Tabledepicting the distribution of patients according to age. 
The common co-morbidities seen were diabetes (26\%) followed by hypertension andIHD( fig 2 ).

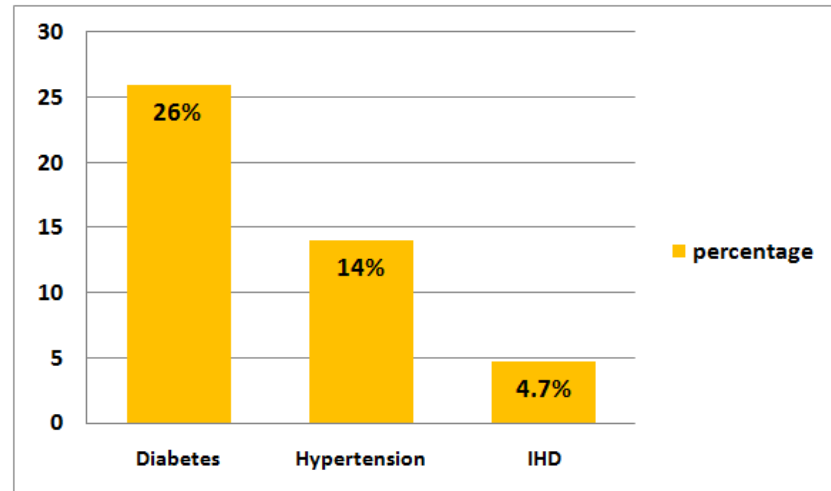

Figure 2. Bar diagram depicting the co-morbidities in the patients

The most common presenting symptom were fever and chills (71.3\%) followed by dysuria (60.7\%).Different classes of drugs used in the treatment of UTI in the study were antibiotics $(99.3 \%, n=149)$, antipyretics ( $99.3 \%$,

$\mathrm{n}=149)$, analgesics $((61.3 \%, \mathrm{n}=92)$. The frequently prescribed antibiotics were Cephalosporins $(82 \%)$ followed byfluroquinolones (64.7).

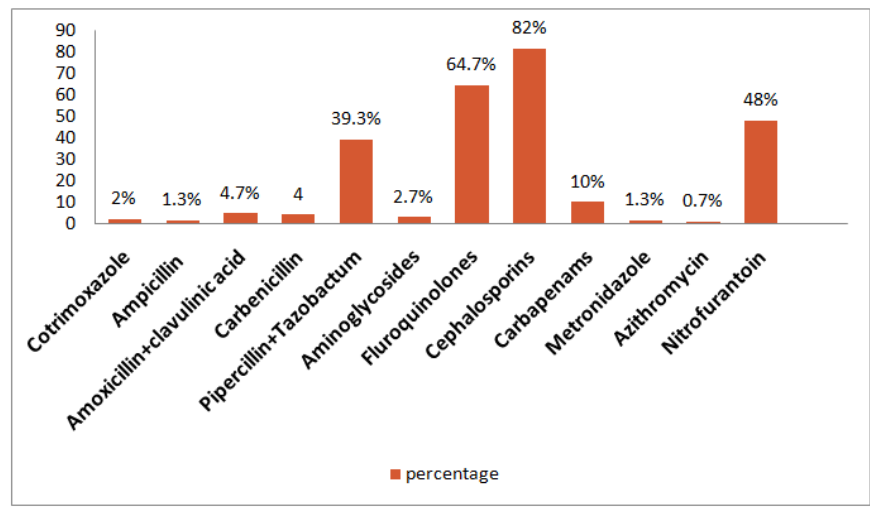

Figure 3: Bar graph depicting the different antibiotics prescribed in the study.

Urine culture and sensitivity test was done in $51.3 \%(\mathrm{n}=77)$ patients and most common pathogens isolated were E.coli (24\%) followed by klebsiella (6.7\%).(Fig 4)

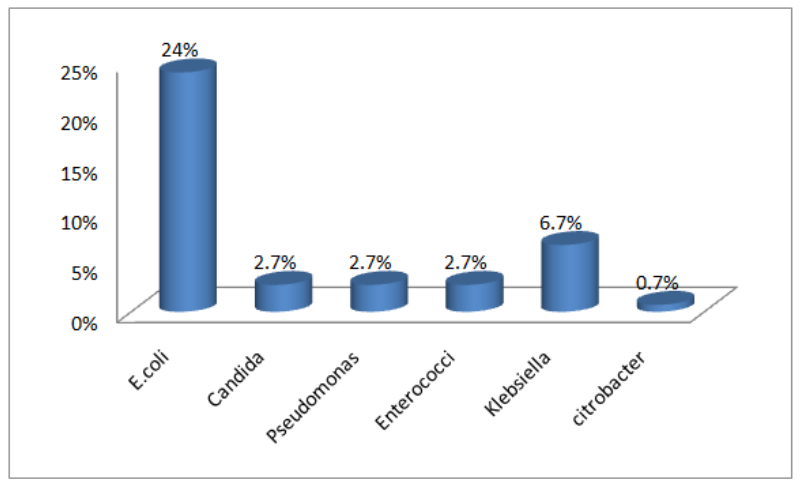

Figure 4. Bar graph depicting the commonuropathogens isolated in the study

A total of 244 potential drug interactions were noted in the study, of which 106 were in pharmacodynamic in nature and 132 were pharmacokinetic in nature.

\begin{tabular}{|c|c|}
\hline CLASS & DRUG INTERACTIONS \\
\hline Pharmacodynamics & 106 \\
\hline Pharmacokinetic & 132 \\
\hline - Absorption & 70 \\
\hline - $\quad$ Distribution & 08 \\
\hline
\end{tabular}




\begin{tabular}{|c|c|c|}
\hline- & Metabolism & 30 \\
\hline- & Elimination & 24 \\
\hline Unkno & & 06 \\
\hline
\end{tabular}

Table 2.Table depicting the types on potential drug interactions.

\section{Discussion}

This was a restrospective observational study conducted in Father Muller Medical College. We assessed the pattern of drugs prescribed, age and gender distribution, most common symptoms, causative pathogens and potential drug interactions.A total of 150 prescriptions were analysed, among which females were about $66 \%$ and males were $34 \%$. This female preponderance was also seen in a study conducted by Dhodi et al ${ }^{5}$ Women are more prone to infections than men because of short urethra. ${ }^{6}$ Majority of the patients belonged to an age group of 19-30 years which was in accordance to a study by Ahamad ${ }^{7}$ where maximum patients were seen in the age group of 21-30 years which are the child bearing age as well as sexually active period. Diabetes mellitus (26\%) was the most common co-morbidity associated with the patients followed by hypertension (14\%) and $\operatorname{IHD}(4.7 \%)$. This finding was comparable to a study by Gorteret al. ${ }^{8}$ The most common presenting symptom were fever and chills $(71.3 \%)$ followed by dysuria $(60.7 \%)$,haematuria $(56.7 \%)$ and pain abdomen $(22.7 \%)$

The total number of drugs prescribed were 1014 in 150 prescriptions and average number of drugs per prescription is 4.46 .About $80.7 \%$ of drugs were prescribed in generic names. This can lead to the lesser cost of therapy. About $78 \%$ of drugs were given by oral route. Antibiotics $(99.3 \%, \mathrm{n}=149)$ were the frequently prescribed class of drug followed by antipyretics( $99.3 \%, \mathrm{n}=149)$ and analgesics $((61.3 \%, \mathrm{n}=92)$. This was similar to a study conducted byMahadevamma et $\mathrm{al}^{9}$ where antibiotics were the first line therapy in the treatment of UTI. Miscellaneous drugs like syrup citralka, proton pump inhibitors, antidiabetics and antihypertensives were also used in the study. Cephalosporins $(82 \%)$ and fluroquinolones $(64.7 \%)$ were the commonly used antibiotics. Similar antibiotic profile was seen in a study by Patel P et al. ${ }^{10}$ Urine culture and sensitivity test was done in about $51.3 \%$ of patients and E.coli was the common pathogen isolated in about $24 \%$ of patients followed by klebsiella in $6.7 \%$ of patients which was similar to a study conducted by Alla $\mathrm{R}$ et al ${ }^{11}$ where E.coli was the predominat pathogen isolated in the study.Total no of potential drug interactions noted were 244 . One hundred and thirty two interactions were pharmacokinetic in nature and 106 interactions were parmacodynamics in nature. Some of the common drug interactions seen were aspirin+albuterol $(n=23)$, ciprofloxacin+ondensetron $(n=3)$, ranitidine +ferrous sulphate $(n=15)$, enalapril +aspirin $(n=9)$.

\section{Conclusion}

Our study revealed that the incidence of UTI was more in females compared to males and majority of the cases were seen in the age group of 19- 30 years. Cephalosporins and fluroquinolones were the commonly prescribed antibiotics. The most common pathogen isolated was E.coli. Majority of potential drug interactions were pharmacokinetic in nature hence periodic auditing of prescriptions is vital for promotion of rational use of drugs, increasing the therapeutic efficacy, cost effectiveness, and minimizing adverse effects and drug interactions.

\section{References}

[1]. ChowtaM.Drug Utilization Pattern in Urinary tract infections: A Retrospective study. Instasci. J. of Med. Sci. \& Clinical Res. 2011; 1(1):13-19.

[2]. Rajat M, Pradeep J, Dass P, Patil S, Gayathri A, Waseem.Drug utilisation study of Urinary Tract Infections in Medicine Department in a Tertiary care Hospital. J Med Dent Sci. 2014 Nov ; 3(63): 13804- 13816.

[3]. Piatkov I, Jones T, McLean M. Drug Interactions, Pharmacogenomics and Cardiovascular Complication, Drug Discovery. Hany ElShemy. 2013

[4]. Shalini, Joshi M, Rashid M, Joshi H. Study of Antibiotic Sensitivity Pattern In Urinary Tract Infection At A Tertiary Hospital. 2016; 2(3): 43-46.

[5]. Dhodi DK, Jaiswar S, Bhagat SB, Gambre RS.A study to evaluate prescribing pattern of antibiotics among patients of urinary tract infection with preexisting renal disorders in a tertiary care hospital. Int J Basic ClinPharmacol. 2014; 3(4): 687-689.

[6]. Shahab HM, Syedumam R, Jebin T, Raja SV. Study of the prescription patterns of medication for urinary tract infection in patients admitted to ICU in a tertiary care hospital.Int J CurrSci .2015;17: 50-55

[7]. Ahmad S. Pattern of urinary tract infection in Kashmir and antimicrobial susceptibility. Bangladesh Med Res Counc Bull 2012; 38 : $79-83$

[8]. Gorter KJ, Hak E, Zuithoff NP, Hoepelman,Rutten GE. Risk of recurrent acute lower urinary tract infections and prescription pattern of antibiotics in women with and without diabetes in primary care. Family Practice, 2010; 0:1-7.

[9]. Mahadevamma L, Krishnagoudar B, Nagar A, Sandeep A.Urinary Tract Infection: Analysis of Prescribing Pattern of Antibiotics. Int J Pharm Sci Res.2012; 3(3):352-357.

[10]. Patel P, Bairy KL, Amberkar MB. A Retrospective Study of Antibiotic Usage among In-Patients of UTI in A Tertiary Care Hospital.Res J Pharm BiolChemSci .2016;7(1):1567-1574.

[11]. Alla R, Kuppast IJ, Mankani KL. A study on prescription pattern of antibiotics for urinary tract infections in shimoga district of Karnataka.Int J Pharm Sci.2012; 3(2): 240 -246. 